\title{
Relación entre sentido de coherencia y adhesión al tratamiento en mujeres adultas con cáncer de mama de un hospital nacional de Lima Metropolitana
}

Relationship between sense of coherence and adhesion to treatment in adult women with breast cancer of a national hospital of Metropolitan Lima

\author{
Rita Selene Orihuela Anaya ${ }^{1}$
}

\section{RESUMEN}

El objetivo fue conocer la relación entre sentido de coherencia (SOC) y adhesión al tratamiento en 100 mujeres adultas con cáncer de mama de un hospital de Lima Metropolitana. Busca identificar también la relación entre el SOC y las variables de conductas de adhesión presentes, motivos, competencias funcionales pasadas y presentes. Se utilizó el cuestionario OLQ29 de Antonovsky y el cuestionario para medir variables psicológicas y conductas de adhesión, adaptado por Mejía. Se encontró una fuerte correlación entre SOC y adhesión al tratamiento $(0,809)$, siendo el SOC predictor de la adhesión $(65,44 \%)$. La variable estadio no representa mayor influencia en la relación que existente entre el SOC y la adhesión al tratamiento

PALABRAS CLAVE: Adhesión al tratamiento, sentido de coherencia, cáncer de mama, mujeres adultas.

\section{SUMMARY}

The objective was to know the relationship between sense of coherence (SOC) and adherence to treatment in 100 adult women with breast cancer from a hospital in Metropolitan Lima. It also seeks to identify the relationship between the SOC and the variables of present adhesion behaviors, motives, past and present functional competences. The questionnaire OLQ29 of Antonovsky and the questionnaire to measure psychological variables and adherence behaviors adapted by Mejía, were used. A strong correlation was found between SOC and treatment adherence (0.809), with SOC being a predictor of adherence $(65.44 \%)$. The stage variable does not represent a greater influence on the relationship between the SOC and treatment adherence.

KEYWORDS: Adherence to treatment, sense of coherence, breast cancer, adult women.

\footnotetext{
${ }^{1}$ Facultad de Psicología, Universidad Peruana Cayetano Heredia. Lima, Perú.

${ }^{a}$ ORCID 0000-0002-7802-6230; Licenciada en Psicología.
} 


\section{INTRODUCCIÓN}

La International Agency for Research on Cancer (IARC, 2012) considera al cáncer de mama como el más frecuente de las neoplasias, seguido del cáncer de próstata y de pulmón. A nivel mundial, el cáncer de mama presenta una incidencia de 43,4 por cada 100 mil habitantes y una mortalidad de 12,9 por cada 100 mil habitantes. Asimismo, en el Perú, según el Instituto Nacional de Enfermedades Neoplásicas (INEN, 2016), organismo que atiende a pacientes con diagnóstico de tumores benignos y malignos, el cáncer de mama ocupa el primer lugar en Lima Metropolitana, con un promedio de 6051 casos nuevos de mujeres diagnosticadas anualmente y 1619 defunciones de pacientes con cáncer de mama. Además, la IARC (2012), menciona que, nuevamente a nivel mundial, entre los 40 a 65 años existe mayor incidencia de cáncer de mama, con 2389 casos nuevos al año. Es en esta etapa también donde se da el inicio de la menopausia, disminución de algunas funciones físicas (visión y audición), inicio de enfermedades cardiovasculares y a nivel psicológico y social existe una tendencia a buscar la realización a nivel profesional y familiar, muchas mujeres establecen sus familias, aunque también surgen cambios en sus roles, divorcio, muerte de los padres, crecimiento de sus hijos a adolescentes o jóvenes, etc.

Por otro lado, si bien se han descrito en cifras la magnitud que tiene el cáncer de mama a nivel mundial, es importante mencionar que los avances en el campo de la salud permiten a las pacientes contar con grandes alternativas de tratamiento como pueden ser procedimientos quirúrgicos, farmacológicos, hormonales y los basados en radiaciones ionizantes que ayudan a la recuperación y control de esa enfermedad. Asimismo, las enfermedades crónicas como el cáncer de mama, requieren un proceso de tratamiento a largo plazo, lo que implica también tener que adaptarse a cambios en el estilo de vida.

Como se ha mencionado, el cáncer de mama considera una serie de procedimientos, que van a ser aplicados dependiendo del tamaño y del estadio del cáncer. Sin embargo, estos procedimientos continúan siendo en muchos casos iatrogénicos y generan altos costos psicológicos y físicos en las pacientes, a pesar de la existencia de avances científicos en la administración de estos tratamientos (Díaz, 2000; Buela-Casal, 1999, citados en Palacios \& Vargas, 2011, p.427). Un claro ejemplo son la quimioterapia y radioterapia que continúan provocando efectos colaterales como náuseas, vómitos, diarrea, mucositis, inmunosupresión, alopecia, astenia, adinamia, etc., interfiriendo con la cotidianidad y el bienestar subjetivo de las pacientes (Fleming, Braddy, Mielszkalski, Cooper \& Cooper, 1996, citados en Palacios \& Vargas, 2011, p. 424). Esto nos permite explicar por qué muchos de los procedimientos que son parte del tratamiento oncológico continúan siendo estigmatizados; los efectos que producen los diversos tratamientos y las experiencias de los propios pacientes refuerzan las etiquetas que se asumen para este tipo de tratamientos, denominándolos como "costosos" y "desagradables"; además, estas etiquetas y prejuicios de la población acerca de los tratamientos, permitirían explicar las respuestas de evitación que tienen las pacientes con diagnóstico de cáncer, ya que son ellas quienes deben decidir si inician o mantienen su tratamiento oncológico (Palacios \& Vargas, 2011, p. 424).

Según las cifras de la Organización Mundial de la Salud (OMS), en los países desarrollados el promedio de adhesión a tratamientos es del $50 \%$, porcentaje que es menor en los países en vía de desarrollo (Sabaté, 2003, citado en Mejía, Piña, Méndez \& Laborin, 2013, p.143). Hoy en día, se evidencia que el porcentaje de adhesión en estudios con pacientes oncológicos oscila entre el $20 \%$ y el $100 \%$, siendo menor el porcentaje de adhesión a tratamientos prolongados como la quimioterapia o radioterapia (Palacios \& Vargas, 2011, p.435). Además, en un estudio reciente en Brasil con pacientes con cáncer de mama bajo tratamiento adyuvante sometido a intervención nutricional educativa, encontraron una baja prevalencia de adhesión a la terapia farmacológica, entre el $28.1 \%$ y el $88.2 \%$ de los usuarios se adhieren al tratamiento (Fritzen, Motter \& Vieira, 2017). Sin embargo, es importante mencionar que en la actualidad son pocas las evidencias en cifras que hablen de la adhesión a tratamientos oncológicos, esto es por la discusión que existe en torno al término de adhesión, lo que dificulta su medición o estudio (Mejía, 2013).

Por ello, es necesario enmarcar esta investigación en el concepto propuesto por Piña y Sánchez (2007), quienes consideran que la adhesión es:

La frecuencia con la que el usuario de los servicios de salud pone en práctica, a lo largo de un periodo variable y a partir de las competencias que dispone, una diversidad de comportamientos que le permiten cumplir de modo eficiente con determinados criterios, en estricta correspondencia con las 
instrucciones provistas por el equipo de salud (p. 401).

De modo que la adhesión constituye el componente final de una secuencia de conductas, que la persona o paciente lleva a cabo una vez recibido el diagnóstico de una enfermedad y además ha sido expuesta a los tratamientos que le corresponden (Sánchez-Sosa, 2002).

En este sentido, de acuerdo a lo planteado por Piña (2012, citado Mejía, 2013):

[...] para poder dar cuenta de por qué y en qué circunstancias una persona practica conductas de adhesión es fundamental disponer de elementos teóricos para así realizar un análisis adecuado y así mismo plantear una medición sistemática de las variables psicológicas que actúan como predictores en dicha práctica [...] (p.28).

En este contexto conceptual, "adhesión al tratamiento" es un constructo multidimensional que envuelve cuatro aspectos: a. competencias pasadas; $b$. competencias presentes; c. motivos y d. conductas de adhesión.

Existen variables psicológicas que afectan a la adhesión al tratamiento, como las actitudes, conocimientos y creencias acerca de la enfermedad y los medicamentos, la percepción de riesgo, la motivación, las experiencias previas, las creencias acerca de la salud y las capacidades para hacer frente a situaciones de estrés como lo sería el recibir un diagnóstico de cáncer de mama (Palacios \& Vargas, 2011, p. 428). Como parte de esas variables se identifica el término Sentido de Coherencia (SOC) en relación con el hecho de que algunas pacientes tienden a afrontar de forma positiva su enfermedad a diferencia de otras. El SOC es definido por Antonovsky (1987) como:

Una orientación global que expresa el grado en el que uno tiene un sentimiento de confianza profundo, generalizado y perdurable, aunque dinámico, de que (1) los estímulos derivados de los medios interno y externo en el curso de la vida son comprensibles, estructurados, predecibles y explicables; (2) uno dispone de recursos para satisfacer las exigencias de estos estímulos, los recursos son manejables y están disponibles con respecto a las demandas que el individuo se va a encontrar derivadas de esos estímulos; y (3) esas demandas son retos, con significado, dignos de implicarse y comprometerse (p. 19).
Antonovsky (1987) se refirió a este término como una disposición relativamente estable que lleva a evaluar las situaciones de la vida como significativas, manejables y predecibles; de este modo, a mayor cantidad de sentido de coherencia, existe mayor posibilidad de bienestar, comprendiendo así a la salud como un continuo, el cual el individuo tiene la tendencia a alcanzar (Antonovsky, 1990); es decir, a mayor fortaleza del sentido de coherencia, mayor es la capacidad de las personas para hacer frente a los estresores psicosociales y así acercarse a la salud dentro del continuo salud-enfermedad. Entonces, el sentido de coherencia podría representar un rol amortiguador frente al diagnóstico y el tratamiento del cáncer de mama, además podría promover que las personas lleven a cabo conductas saludables que fortalezcan su salud.

En este contexto, Torres (2014), encontró en pacientes con cáncer de mama que pertenecen a la adultez media, una relación directa entre el SOC y el soporte social que reciben. Según el Modelo Salutogénico, el soporte constituye ser uno de los recursos generales de resistencia externos más importantes para el desarrollo de un fuerte SOC (Antonovsky, 1987). En el caso de las pacientes que padecen cáncer de mama, el soporte social fortalece y promueve su SOC pues disminuye la preocupación por el progreso de la enfermedad, así como los sentimientos de culpa y vergüenza, entre otros malestares (Jeffe, Liu, Schootman \& Waters, 2013). Dentro de estos beneficios que brinda el soporte social, se encuentra menos ansiedad de muerte en pacientes oncológicos que cuentan con este recurso (Adelbratt \& Strang, 2000; Gonen et al., 2012; Jo \& Song, 2012).

Las consideraciones antes expuestas nos llevan a plantear la siguiente interrogante ¿Qué relación existe entre el SOC y adhesión al tratamiento en mujeres adultas con cáncer de mama de un Hospital Nacional de Lima Metropolitana?

El objetivo general de la presente investigación es conocer la relación que existe entre el SOC y la adhesión al tratamiento en mujeres adultas con cáncer de mama de un Hospital Nacional de Lima Metropolitana. Como se objetivos específicos se plantea lo siguiente:

1. Describir las conductas de adhesión presentes en mujeres adultas con cáncer de mama de un Hospital Nacional de Lima Metropolitana.

2. Describir los niveles del SOC en mujeres adultas con cáncer de mama de un Hospital Nacional de Lima Metropolitana.

3. Identificar la relación entre el sentido de coherencia 
y las conductas de adhesión presentes en mujeres adultas con cáncer de mama de un Hospital Nacional de Lima Metropolitana.

4. Identificar la relación entre el SOC y las competencias funcionales pasadas en mujeres adultas con cáncer de mama de un Hospital Nacional de Lima Metropolitana.

5. Identificar la relación entre el SOC y las competencias funcionales presentes en mujeres adultas con cáncer de mama de un Hospital Nacional de Lima Metropolitana.

6. Identificar la relación entre el SOC y la dimensión "motivos subyacentes para cumplir o no las conductas de adhesión", en mujeres adultas con cáncer de mama de un Hospital Nacional de Lima Metropolitana.

7. Identificar el valor predictivo del SOC en la adhesión al tratamiento en mujeres adultas con cáncer de mama de un Hospital Nacional de Lima Metropolitana.

8. Identificar la relación que existe entre el SOC y adhesión al tratamiento en mujeres adultas con cáncer de mama de un Hospital Nacional de Lima Metropolitana, según el tipo de tratamiento.

9. Identificar la relación que existe entre el SOC y adhesión al tratamiento en mujeres adultas de un Hospital Nacional de Lima Metropolitana, según el estadio de enfermedad.

\section{MATERIAL Y METODOS}

Participaron 100 pacientes mujeres diagnosticadas con cáncer de mama que acudían a un hospital de Lima Metropolitana durante los meses de Diciembre del 2015 a Abril del 2016. La edad promedio de las pacientes fue de 51 años ( \pm 7 años) en un rango de 40 a 65 años. El 51\% era casada, el 20\% soltera, el 14\% viuda, $12 \%$ divorciada y sólo $3 \%$ era conviviente. El $5 \%$ no tenía hijos, $65 \%$ de uno a dos hijos, y el $30 \%$ de 3 a 7 hijos. Al momento de la evaluación, todas se encontraban orientadas en tiempo, espacio y persona. En lo que respecta a las características clínicas, la frecuencia del cáncer en la mama fue prácticamente la misma en la mama derecha (51\%) que en la izquierda $(49 \%)$. Solamente la quinta parte de mujeres seguían un tratamiento con terapia hormonal, pero todas $\sin$ excepción eran tratadas con cirugía y quimioterapia; $\sin$ embargo, al 76\% también se le trató con radioterapia. El tiempo promedio transcurrido desde el diagnóstico fue de 2,2 años en un rango de 1 a 5 años, y similarmente el tiempo trascurrido desde la cirugía fue de 2,1 años en un rango de 1 a 5 años. El estadio de la enfermedad más frecuente fue el II-B, seguido del
II-A con frecuencias de $41 \%$ y $27 \%$ respectivamente. Una sola paciente estuvo en el estadio IV y otra en el estadio III-C.

El tipo de muestreo utilizado fue no probabilístico de tipo accidental o por conveniencia, donde se eligió a los pacientes de acuerdo a los criterios de inclusión. El tamaño de la muestra fue obtenida a través de la siguiente fórmula, la cual es aplicada cuando los objetivos a determinar son únicamente de correlación:

$n=\left(\frac{z_{1-\sigma / 2}+z_{1-\rho}}{\frac{1}{2} \ln \left(\frac{1+\gamma}{1-\gamma}\right)}\right)^{2}+3$

Para el cálculo se tuvo en cuenta una seguridad del $95 \%$ y un poder estadístico de $80 \%$. Se obtuvo una $\mathrm{n}=85$; sin embargo, finalmente, la muestra se aumentó a 100 .

\section{Instrumentos}

\section{Ficha de datos.}

Recoge los datos sociodemográficos y clínicos de las pacientes como edad, estado civil, número de hijos, lugar de procedencia, historia clínica, diagnóstico, tiempo de enfermedad y tiempo y tipo de tratamiento.

Cuestionario de Orientación a la Vida (Antonovsky, 1987)

Mide el SOC, constructo que se relaciona con el afrontamiento de situaciones traumáticas y que se ha empleado como predictor de medidas de salud percibida y objetiva. Este constructo se considera como relacionado a orientación a la vida (Antonovsky, 1987); consta de 29 ítems con respuestas en escala Likert de 1 a 7 puntos, donde 1 indica "nunca tuve este sentimiento", mientras que 7 indica "siempre tuve este sentimiento". Este instrumento tiene tres escalas, cuyos alfas son los siguientes: Comprensibilidad $(0,80)$, sentido emocional a la vida $(0,82)$ y manejabilidad $(0,80)$. La correlación test-retest fue de 0,85 (Antonovsky, 1987). Arévalo (2007) realizó una traducción al español y adaptación en mujeres peruanas en el marco de un estudio acerca del estrés y pobreza en dicha población. En este estudio, se obtuvo una estructura unifactorial y un alfa de .80 . Posteriormente, Saravia (2011) realizó un estudio en el Perú con pacientes limítrofes y estudiantes de ingeniería, obteniendo un alfa de .88 en el grupo de pacientes y de 0,90 en el de estudiantes. 
Cuestionario de variables psicológicas y conductas de adhesión al tratamiento oncológico por cáncer de mama.

Este instrumento es una adaptación del cuestionario de variables psicológicas y conductas de adherencia al tratamiento en personas seropositivas (VPAD-24). Dicha adaptación a pacientes oncológicas del Perú fue realizada por Mejía, Piña, Méndez y Laborín (2013). La adaptación presenta un alfa de 0,70 , lo que sugiere una confiabilidad aceptable del instrumento (Mejía et al., 2013). Este cuestionario cuenta con tres factores con valores propios superiores a 1 , que explican el 45,6\% de la varianza: Competencias Pasadas; Motivos y Competencias Presentes; entre éstas últimas, algunas están relacionadas con la adhesión al tratamiento y otras son de tipo social y personal (Mejía et al., 2013). El instrumento cuenta además con ítems que evalúan conductas de adhesión al tratamiento.

\section{Procedimiento}

El procedimiento comenzó con el trámite administrativo para llevar a cabo la investigación en el hospital seleccionado de Lima Metropolitana. Se coordinó con el jefe del consultorio externo de mamas y tejidos blandos. Posteriormente, se identificó a las participantes a través de las listas de atenciones en el sistema, utilizando los criterios de atención. El horario en el que se aplicaron las pruebas, fue martes y jueves desde las 6 am hasta las 9 a.m. La aplicación de las pruebas se realizó de manera individual, la primera información brindada fue el consentimiento informado en el que se recalcó que la participación en esta investigación era voluntaria, así como los objetivos y la existencia de la confidencialidad en los datos a través de la codificación. Luego de la obtención del consentimiento informado se solicitó los datos demográficos de cada participante. Posteriormente, se procedió a brindar las indicaciones sobre los instrumentos, en primer lugar, el cuestionario de variables psicológicas y adherencia al tratamiento y luego, la escala de SOC. En ambos casos, cada ítem fue leído por la investigadora a la paciente.

\section{Análisis de datos}

Los datos fueron analizados con el software estadístico SPSS-IBM 21. En la parte descriptiva se calcularon las frecuencias de las conductas de adhesión. Para las correlaciones, primero se identificó el tipo de correlación que mejor describiría a las dos variables estudiadas a partir del gráfico de dispersión, los cuales arrojaron relaciones no lineales, por lo que se calcularon correlaciones eta. Para identificar la predicción, se elevó al cuadrado el coeficiente de correlación eta.

\section{RESULTADOS}

Se halló que de las 100 pacientes, el 53\% reportó practicar siempre comportamientos relacionados a asistir a sus consultas médicas, el $92 \%$ reportó asistir a sus sesiones de tratamiento para quimioterapia y/o radioterapia $\mathrm{y} / \mathrm{u}$ otro tratamiento. Por el contrario, las siguientes conductas, tienen un menor porcentaje de adhesión, el 49\% de pacientes reportan practicar siempre comportamientos relacionados a seguir una dieta recomendada, el 31\% realiza siempre los ejercicios propios para su rehabilitación y sólo el $35 \%$ cuida siempre las zonas de su cuerpo. Además, se encontró que del total de conductas de adhesión recomendadas como parte del tratamiento oncológico por cáncer de mama, el $6 \%$ de personas practicaron siempre las cinco conductas recomendadas, en tanto que el 94\% nunca practicó al menos una de estas conductas o dejó de practicarlas (tabla 1).

Respecto de los niveles de SOC en las pacientes investigadas se identificó dos grupos: pacientes con sentido en un nivel regular (57\%) y pacientes con sentido positivo (43\%), es decir, no hubo pacientes con sentido negativo de coherencia.

La correlación eta entre SOC y adhesión al tratamiento fue de .809. Entonces, se espera que a mayor SOC, mayor adhesión al tratamiento oncológico. Para identificar el valor predictivo del SOC en la adhesión al tratamiento oncológico, se obtuvo el coeficiente de correlación eta al cuadrado, dando como resultado el valor 0,654 . Es así que podemos decir que el $65,4 \%$ de la adhesión al tratamiento puede ser predicha a partir de la variable SOC. El valor eta cuadrado $(0,6544)$ indica que el $65,44 \%$ de la varianza en la adhesión al tratamiento se debe a la varianza en SOC. La tabla 2 muestra además, las correlaciones entre el SOC y las variables psicológicas de la adhesión. Tal como se aprecia, todas las correlaciones fueron positivas y relativamente altas, lo que indica que a un mayor SOC se esperaría mejores competencias funcionales pasadas y presentes y mejores motivos para su adhesión al tratamiento (tabla 3). 
Tabla 1: Conductas de adhesión presentes

\begin{tabular}{|c|c|c|c|c|c|}
\hline \multicolumn{2}{|c|}{ Ítems } & \multirow{2}{*}{$\begin{array}{c}\text { Nunca } \\
0 \%\end{array}$} & \multirow{2}{*}{$\begin{array}{l}\begin{array}{l}\text { Pocas } \\
\text { Veces }\end{array} \\
8 \%\end{array}$} & \multirow{2}{*}{$\begin{array}{l}\begin{array}{l}\text { Muchas } \\
\text { Veces }\end{array} \\
39 \%\end{array}$} & \multirow{2}{*}{$\begin{array}{r}\text { Siempre } \\
53 \%\end{array}$} \\
\hline 6 & $\begin{array}{l}\text { ¿Asiste a las consultas programadas o el personal de } \\
\text { salud para revisar y/o analizar su tratamiento? }\end{array}$ & & & & \\
\hline 7 & $\begin{array}{l}\text { ¿Asiste a sus citas para recibir quimioterapia, } \\
\text { radioterapia, en la fecha programada? }\end{array}$ & $0 \%$ & $4 \%$ & $4 \%$ & $92 \%$ \\
\hline 8 & $\begin{array}{l}\text { ¿Incluye en su dieta diaria alimentos recomendados por } \\
\text { su médico o nutricionista? }\end{array}$ & $0 \%$ & $8 \%$ & $43 \%$ & $49 \%$ \\
\hline 9 & $\begin{array}{l}\text { ¿Realiza los ejercicios físicos recomendados por los } \\
\text { especialistas para su rehabilitación? }\end{array}$ & $0 \%$ & $15 \%$ & $54 \%$ & $31 \%$ \\
\hline 10 & $\begin{array}{l}\text { ¿Cuida las zonas afectadas por la enfermedad a la } \\
\text { exposición de calor (cocina, plancha, sol, etc.)? }\end{array}$ & $2 \%$ & $20 \%$ & $43 \%$ & $35 \%$ \\
\hline \multicolumn{2}{|c|}{ En General } & $0,4 \%$ & $11,0 \%$ & $36,6 \%$ & $52,0 \%$ \\
\hline
\end{tabular}

Tabla 2: Correlaciones Eta del SOC con variables psicológicas de adhesión

\begin{tabular}{cc}
\hline Variables & eta \\
\hline Competencias pasadas & 0,741 \\
Competencias presentes & 0,769 \\
Motivos & 0,855 \\
Conductas de adhesión presentes & 0,749 \\
\hline
\end{tabular}

Tabla 3: Sentido de coherencia y adhesión, controlando la variable tipo de tratamiento

\begin{tabular}{llllc}
\hline Tratamiento & & & & Correlación \\
\hline Sin hormonas & Nominal por intervalo & Eta & Adh. Tto. dependiente & 0,777 \\
Con Hormonas & Nominal por intervalo & Eta & Adh. Tto. dependiente & 0,687 \\
Total & Nominal por intervalo & Eta & Adh. Tto. dependiente & 0,709 \\
\hline
\end{tabular}

Tabla 4: Sentido de coherencia y adhesión al tratamiento, controlando la variable estadio de la enfermedad

\begin{tabular}{llllc}
\hline Estadio & & & & Correlación \\
\hline IA, IB & Nominal por intervalo & Eta & Adh. Tto. dependiente & 0,887 \\
IIA, IIB & Nominal por intervalo & Eta & Adh. Tto. dependiente & 0,725 \\
III, IV & Nominal por intervalo & Eta & Adh. Tto. dependiente & 0,850 \\
Total & Nominal por intervalo & Eta & Adh. Tto. dependiente & 0,709 \\
\hline
\end{tabular}

Se identificó la relación entre el SOC y la adhesión al tratamiento controlando la variable tipo de tratamiento. Los resultados obtenidos controlando la variable tipo de tratamiento indican relaciones significativas entre SOC y adhesión al tratamiento; es decir que la relación entre ambas variables no se ve afectada por el tipo tratamiento que reciben las pacientes (tabla 3 ).
Finalmente, se identificó la relación entre el SOC y la adhesión al tratamiento controlando la variable estadio de la enfermedad. Para un mejor análisis y control de la variable estadio, se dividió a la población en tres grupos, pacientes en estadios IA y IB, pacientes en estadios IIA y IIB y pacientes en estadios III y IV. En la tabla 4 se muestran las correlaciones respectivas, notándose que las pacientes con estadio IA y IB 
presentan la correlación más alta en tanto que la más baja corresponde a las pacientes en los estados IIA y IIB, sin embargo, las diferencias entre los grupos no son significativas.

\section{DISCUSIÓN}

El objetivo general fue conocer la relación que existe entre el SOC y la adhesión al tratamiento en mujeres adultas con cáncer de mama. Los resultados indican una fuerte correlación de .809 , es así que a mayor SOC se espera que las pacientes cuenten con una mejor adhesión al tratamiento. Los resultados indican también que el 65,44\% de la varianza en la adhesión al tratamiento puede ser predicha a través de la varianza en el SOC. Esta relación nos brinda la posibilidad de atender oportunamente a aquellos pacientes que tengan un nivel regular o bajo de SOC, ya que serían éstas las pacientes más propensas a no presentar una buena adhesión a su tratamiento o a descontinuarlo, afectando considerablemente su calidad de vida. Estos resultados son similares a los reportados por Moya (2014) en el Perú, quien estudió la relación entre SOC y adhesión al tratamiento antirretroviral, hallando una relación mediana significativa entre estas dos variables $(\mathrm{r}=0,43)$. Además, según la teoría planteada por Antonovsky (1987), las personas con SOC alto tienen una mayor probabilidad de adoptar conductas saludables y poseen un nivel de salud autorreferido más estable ante acontecimientos vitales de carácter estresante (Richardson y Ratner, 2005). Todo ello nos permitiría señalar que las pacientes con cáncer de mama con un SOC más alto tienden a mostrar características más saludables desde el punto de vista físico y psicológico incluyendo el contar con competencias (conocimientos habilidades y destrezas) para llevar a cabo conductas de autocuidado, y además considerar que dichas conductas son pertinentes y coherentes con el tratamiento y contribuyen con su percepción de salud.

Sin embargo, a pesar de lo anterior, se halló que no todas las conductas de adhesión son practicadas con igual frecuencia. Por ejemplo, el 53\% refieren asistir "siempre" a consulta médica y el $92 \%$ reciben "siempre" tratamiento de quimioterapia, radioterapia o terapia hormonal. Mientras que un $49 \%$ de las pacientes refieren seguir "siempre" un dieta recomendada, $31 \%$ realizan "siempre" ejercicios físicos para la rehabilitación del brazo y 35\% cuidan "siempre" las zonas afectadas de la exposición al calor. Es decir, las pacientes tienden a asistir a sus consultas y tratamientos, pero en cambio, están menos propensas a cumplir con las recomendaciones recibidas del personal de salud. Estos resultados son similares a los de Mejía (2013), quien identificó que las practicas más frecuentes en pacientes con cáncer de mama corresponden a asistir a citas médicas $(83 \%)$, asistir a tratamientos como la quimioterapia y radioterapia (93\%) y en menor porcentaje seguir dieta balanceada $(53 \%)$ y realizar ejercicios (34\%) y cuidado de zonas afectadas (40\%). Otras investigaciones que contribuyen con lo que se ha encontrado en esta investigación, es de los autores Alayón y Mosquera (2008) quienes estudiaron a una muestra de pacientes diabéticas, donde encontraron bajas tasas de adhesión en: realizar ejercicios físicos, seguir dieta balanceada y en el cuidado de miembros inferiores. De un modo semejante Torres y Piña (2010), con pacientes de VIH, reportaron porcentajes bajos en la práctica de ejercicios físicos $(31 \%)$ y seguir dieta recomendada (37\%), en tanto que el consumo de medicamento alcanzó porcentajes elevados de adhesión (89\%). Todas estas investigaciones, incluido lo encontrado en la presente, nos alerta sobre la complejidad en la ejecución de conductas de adhesión vinculadas al cambio del estilo de vida (alimentación, ejercicios y cuidados) cuya importancia es igual a la del consumo de medicamentos, ya que al no cumplirlas generan otras afecciones como celulitis en las zona próximas donde se intervino quirúrgicamente; por ejemplo, en pacientes que no cuidan las zonas afectadas o no realizan los ejercicios indicados, requiriendo así mayor intervención. Por otro lado, Ortiz y Ortiz (2007) realizaron una revisión de la literatura sobre la adherencia terapéutica en diferentes enfermedades crónicas y agudas y mencionan que las tasas son diferentes de una patología aguda versus una crónica. Estos autores, también refieren que, cuando el tratamiento de la enfermedad consiste en un patrón complejo de conductas e invaden diferentes aspectos del paciente, se afecta la adhesión terapéutica, por lo que podría establecerse una relación entre mayor complejidad del tratamiento, menos adherencia terapéutica.

Se encontró que el $57 \%$ de las pacientes cuenta con un sentido regular y el $43 \%$ un sentido alto o positivo, de manera que no se identificó a ninguna paciente con un SOC negativo. Papalia, Olds y Feldman (2010), al describir las características de la adultez media, refieren que en esta etapa las personas se encuentran más preparadas para afrontar situaciones de estrés, en comparación de otros grupos de edad; debido a que poseen mejor percepción de lo que quieren hacer para cambiar las situaciones problemáticas y probablemente son más capaces de 
aceptar aquellas cosas que no se pueden cambiar. En la muestra de la presente investigación, las mujeres adultas presentaron puntuaciones más altas en ítems que corresponden al soporte que reciben de su entorno y a la percepción positiva de su vida en el presente y en el futuro. Torres (2014) en su investigación con pacientes con cáncer de mama en Lima, encontró que sólo las mujeres mayores de 45 años perciben como positivo el soporte que reciben y menciona que en el caso de las pacientes que padecen cáncer de mama, el soporte social fortalece y promueve su SOC pues disminuye la preocupación por el progreso de la enfermedad, así como los sentimientos de culpa y vergüenza, entre otros malestares (Torres, 2014). En general, estos hallazgos están en línea con lo señalado por Antonovsky (1987), en el sentido de que el soporte social es uno de los recursos generales de resistencia externos más importantes para el desarrollo de un fuerte SOC.

Se halló también una correlación de .741 entre el SOC y las competencias funcionales pasadas, de modo que a mayor SOC se esperaría que las personas hayan puesto en práctica sus competencias pasadas de forma óptima. Algunos estudios previos tienen relación con este resultado. Por ejemplo, Rohani, Abedi, Sundberg y Langius-Eklof (2015) realizaron un estudio longitudinal del SOC en pacientes con cáncer de mama, y encontraron que el SOC sirvió como mediador en tres dimensiones: calidad de vida, funcionamiento emocional y salud mental, tanto antes del diagnóstico de cáncer de mama como después del mismo. Esto quiere decir que dichas pacientes adoptaron conductas que fortalecían y fortalecen su salud mental y calidad de vida incluso antes de su diagnóstico de cáncer de mama, lo que se relacionaría con las competencias pasadas. Pero ese patrón conductual se mantuvo incluso seis meses después de su diagnóstico, lo que es similar a las competencias presentes, lo que guarda también relación con los resultados del presente estudio, en este caso, la correlación de .769 entre SOC y competencias presentes. Por tanto, a mayor SOC se esperaría que los pacientes pongan en práctica sus competencias después del diagnóstico de cáncer. Rohani et al., (2015) concluyeron que un mayor SOC como recurso interno puede servir como un factor psicológico protector en el proceso de adaptación de los pacientes a un diagnóstico médico, ya sea antes o después del diagnóstico de cáncer.

Se obtuvo una fuerte correlación (.855) entre SOC y motivos para ejecutar o no las conductas de adhesión al tratamiento. Según Piña y Sánchez (2007), una persona cuenta con "motivos", cuando está propensa a realizar algo siempre y cuando lo quiera llevar a cabo porque lo considera oportuno y pertinente y esto se ve reforzado con la obtención de consecuencias positivas como recompensas o expresiones verbales. Esta relación con el SOC, puede estar asociado a uno de sus tres componentes: el sentido emocional a la vida, siendo esta la habilidad de la persona para dar sentido efectivo a la vida, entendiéndose como la capacidad para invertir su energía en los problemas que existen en el ambiente, dándole un significado a las situaciones y percibiéndolas como retos (Antonovsky, 1990). Además, éste es el componente más importante, ya que sin un componente argumentativo que motive, los otros dos componentes del SOC: comprensibilidad y manejabilidad se vuelven temporales. Esto podemos relacionarlo con lo encontrado en la investigación donde el mayor motivo de las pacientes es el deseo de estar bien de salud el mayor tiempo posible (92\%), seguido de experimentar beneficios en su salud (91\%), que son refuerzos que posibilitan que lleven a cabo sus prácticas de adhesión al tratamiento. Es importante mencionar, que no se han encontrado investigaciones que estudien ambas variables.

También se halló una fuerte relación (.849) entre el SOC y las conductas de adhesión presentes, es decir aquellas conductas que son parte del tratamiento de cáncer de mama. Antonovsky (1987) ya había mencionado que las personas con SOC alto tienen una mayor probabilidad de llevar a cabo conductas saludables. Algunos datos indican que un SOC alto está asociado con modos de actuar que pueden favorecer la práctica de conductas saludables, Por ejemplo, Sarenmalm, Browall, Persson, Dickson y Gaston (2013) encontraron que las pacientes con cáncer de mama con un SOC alto presentaban mejores estrategias de afrontamiento de su enfermedad, así como mejor estado de salud y calidad de vida, concluyendo que la escala SOC puede ser una herramienta de detección útil para identificar individuos particularmente vulnerables a la angustia e incapaces de lidiar adecuadamente con su enfermedad, como lo sería poner en practica conductas saludables.

En el grupo de no tratamiento hormonal" (recibieron quimioterapia, radioterapia y cirugía) se obtuvo una relación de 0,777 entre el SOC y la adhesión, mientras que en el grupo de tratamiento hormonal (recibieron quimioterapia, radioterapia, hormonoterapia y cirugía), se encontró una relación de 0,687. Sin embargo, las diferencias entre estas correlaciones no son significativas. Es decir, el recibir o no tratamiento 
hormonal no modifica la relación entre el SOC y la adhesión, si bien la correlación algo más baja en el grupo de tratamiento hormonal indicaría que para el mismo nivel de SOC la adhesión es también algo más baja. Probablemente este efecto se deba a los efectos secundarios que implica el tratamiento hormonal, según Grunfeld, Hunter, Sikka y Mittal, citados por Palacios \& Vargas, (2011), quienes realizaron una revisión de la literatura sobre la adhesión a la quimioterapia y radioterapia en pacientes oncológicos, y determinaron que el motivo principal que tienen las pacientes para no tomar el Tamoxifeno son los efectos secundarios, tales como síntomas urinarios, cansancio, sofocos, sudores nocturnos, entre otros. En nuestra muestra el $21 \%$ de las pacientes consume anastrozol y el 14\% tamoxifeno, entre otros, los cuales tienen efectos secundarios no solo a nivel físico sino también emocional.

Finalmente, se encontró que el SOC se correlaciona con mayor fuerza a la variable adhesión al tratamiento en pacientes con estadio IA y IB $(0,887)$, seguido de pacientes en los estadios III, IV $(0,850)$ y por último las pacientes en el estadio IIA y IIB $(0,725)$. Es según estos resultados que podemos concluir que la variable estadio no representa mayor influencia en la relación que existente entre el SOC y la adhesión al tratamiento. No se han encontrado investigaciones referentes a la influencia de los estadios; sin embargo, en su mayoría las pacientes de esta investigación no contaban con información exacta acerca del estadio en el que se encontraban, esto podría influenciar en que esta variable no sea significativa a la hora de relacionar SOC y adhesión al tratamiento.

\section{Correspondencia:}

Rita Selene Orihuela Anaya

Correo electrónico: rita.orihuela@upch.pe

La autora declara que no tiene conflictos de interés. La presente investigación ha sido financiada por la propia autora y no es parte de un proyecto mayor.

\section{REFERENCIAS BIBLIOGRAFICAS}

Adelbratt, S. \& Strang, P. (2000). Death anxiety in brain tumorpatients and their spouses. Palliative Medicine, 14, 499-507.

Alayón, A. \& Mosquera, M. (2008). Adherencia al tratamiento basada en comportamientos en pacientes diabéticos. Revista de Salud Pública, 10, 777-787. Recuperado desde http://www.scielosp.org/pdf/rsap/ v10n5/v10n5a10.pdf

Antonovsky, A. (1987). Unraveling the mystery of health: How people manage stress and stay well. San Francisco: Jossey-Bass.

Antonovsky, A. (1990). Pathways leading to succesful coping and health. En M. Rosenbaum (Ed.), Learned resourcefulness: On coping skills, self-control, and adaptative behaviour. Nueva York: Springer.

Arévalo, M.V. (2007). Stress and extreme poverty in Peruvian women. Tesis doctoral. Nijmegen, Países Bajos: Radboud University.

Fernandez, M. (2009). Estrés percibido, estrategias de afrontamiento $y$ sentido de coherencia en estudiantes de enfermería: su asociación con salud psicológica y estabilidad emocional. (Tesis doctoral. Universidad de León, Leon, España).

Fritzen, J., Motter, F. R. \& Paniz, V. M. (2017). Regular access and adherence to medications of the specialized component of pharmaceutical services. Revista de Saúde Pública, 51, 109.

Gonen, G., Kaymak, S., Cankurtaran, E., Karslioglu, E., Ozalp E. \& Soygur, H. (2012). The factors contributing to death anxiety in cancer patients. Journal of Psychosocial Oncology, 30, 347-358.

Instituto Nacional de Enfermedades Neoplásicas (2016). Perfil epidemiológico. Lima: Instituto Nacional de Enfermedades Neoplásicas. Recuperado De: http://www.inen.sld.pe/portal/estadisticas/datosepidemiologicos.html

International Agency for Research on Cancer (2012). Cancer Incidence and Mortality Worldwide. Washington: International Agency for Research on Cancer. Recuperado de: http://globocan.iarc.fr/ Pages/fact_sheets_population.aspx

Jeffe, D. , Waters, E., Liu, Y. \& Schootman, M. (2013). Worry about cancer progression and low perceived social support: Implications for quality of life among early-stage breast cancer patients. Annals of Behavioral Medicine, 45, 57-68.

Jo, K. \& Song, B. (2012). Effect of family cohesion, subjective happiness and other factors on death anxiety in Korean elders. Journal of Korean Academy of Nursing, 42, 680-688.

Mejía R., Piña, A., Méndez J. \& Laborín J. (2013). Versión peruana de un cuestionario que mide variables psicológicas relacionadas con la adhesión en mujeres con cáncer de mama. Psicooncología, 10, 419-453. DOI:https://doi.org/10.5209/rev_PSIC.2013.v10.41953 Mejía, R. (2013). Variables Psicológicas y conductuales de adhesión al tratamiento oncológico en pacientes con cáncer de mama. Lima: Universidad Nacional Federico Villarreal.

Moya, M. C. (2014). Sentido de coherencia y adhesión terapéutica antirretroviral en personas con VIH Sida. (Tesis de licenciatura. Pontificia Universidad Católica del Perú, Lima, Perú). 
Ortiz, M. \& Ortiz, E. (2007). Psicología de la salud: Una clave para comprender el fenómeno de la adherencia terapéutica. Revista Médica de Chile, 135, 647-652.

Palacios, X. \& Vargas, L. (2011). Adherencia a la quimioterapia y radioterapia en pacientes oncológicos: Una revisión de la literatura. Psicconcología, 8, 2-3. Recuperado de: http://revistas.ucm.es/index.php/PSIC/ article/view/37890

Papalia, D. E., Olds, S. W. \& Feldman, R. D. (2010). Desarrollo humano. Ciudad de Mexico: McGraw-Hill.

Piña, J. \& Sánchez, J. (2007). Modelo psicológico para la investigación de los comportamientos de adhesión en personas con VIH. Universitas Psychológica, 6, 399-407. Recuperado desde http://redalyc.uaemex.mx/src/inicio/ ArtPdfRed.jsp?iCve $=64760217$

Richardson, C. G. \& Ratner. P.A. (2005). Sense of coherence as a moderator of the effects of stressful life events on health. Journal of Epidemiology and Community Health, 59, 979-984.

Rohani, C., Abedi, H.-A., Sundberg, K. \& LangiusEklöf, A. (2015). Sense of coherence as a mediator of health-related quality of life dimensions in patients with breast cancer: a longitudinal study with prospective design. Health and Quality of Life Outcomes,
13, 195. doi: http://doi.org/10.1186/s12955-015-0392-4 Sánchez-Sosa, J. J. (2002). Treatment adherence: The role of behavioural mechanism and some implications for health care interventions. Revista Mexicana de Psicología, 19, 85-92.

Saravia, J. (2011). Sentido de Coherencia en pacientes limitrofes y estudiantes de ingeniería. (Tesis de Licenciatura. Pontificia Universidad Católica del Perú, Lima, Perú).

Sarenmalm, E., Browall, M., Persson, L., Dickson, J. \& Gaston, F. (2013). Relationship of sense of coherence to stressful events, coping strategies, health status, and quality of life in women with breast cancer. Psychooncology, 22(1), 20-27. doi: 10.1002/ pon. 2053

Torres, A. \& Piña, J. (2010). Asociación entre variable psicológicas y sociales con la adhesión en personas con diabetes tipo 2. Terapia Psicológica, 28, 45-53. Recuperado de http://wwwscielo.cl/scielo.php?script= sci_arttext\&pid=S0718-48082010000100005

Torres, B. (2014). Sentido de Coherencia y Ansiedad - rasgo en una muestra de pacientes que padecen cáncer de mama. (Tesis de pregrado. Pontificia Universidad Católica del Perú, Lima, Perú). 